\title{
Hearing aid through skin sensory for profound deaf people
}

\section{[version 1; peer review: 1 approved, 1 not approved]}

\author{
Yasothei Suppiah (D1), M Chandran Maruthan², Fazly Salleh Abas \\ ${ }^{1}$ Faculty of Engineering \& Technology, Multimedia University, Jalan Ayer Keroh Lama, Melaka, 75450 Bukit Beruang, Malaysia \\ ${ }^{2}$ Department of Electrical Engineering, Politeknik Melaka, No.2, Jalan PPM 10, Plaza Pandan Malim, Melaka, 75250, Malaysia
}

V1 First published: 15 Sep 2021, 10:926

https://doi.org/10.12688/f1000research.72979.1

Latest published: 15 Sep 2021, 10:926

https://doi.org/10.12688/f1000research.72979.1

\section{Abstract}

An individual with profound deafness or total hearing loss has a hearing threshold of $80 \mathrm{~dB}$ or more. The ineffectiveness of hearing aids, surging costs and complex surgeries for cochlear implants have discouraged many to opt for these types of treatments. Hence, this research aims to provide an alternative hearing aid that stimulates "hearing" through the skin sensory, which is more affordable and accessible for the profoundly deaf or total hearing loss community. We have developed four initial vibrating transducers with single spectrum, which are strapped to a belt. The transducers pick up audible sounds through a microphone, amplifies the sound to a highlevel signal, stimulating a vibration pattern on the human skin sensory. The belt was tested on 30 random people who identified as normal, partial, and profoundly deaf. When the belt was strapped to the individual's waist, audible sound was played (stimulus) and the individual was asked whether he/she can feel a stimulation or vibration on their skin, and if so, state the sound source direction. Based on the test, all individuals were able to feel the vibrating stimulation on their skin, and they were also able to state the directions accurately. The various vibrating pattern that stimulates the human sensory system for the profoundly deaf can be learned over time, which could serve as useful information. However, interpreting and identifying the different types of vibrating pattern perceived through the skin remains a huge challenge for profoundly deaf people. As hearing through skin sensory is a very new area of research, there are very limited research articles published in this field. Thus far, this is the first study to evaluate the method of audio spectrum to develop hearing aid through skin sensory.

\section{Keywords}

profound deaf, sound, hearing device, vibrating pattern, skin sensory, stimulation, transducers, spectrum

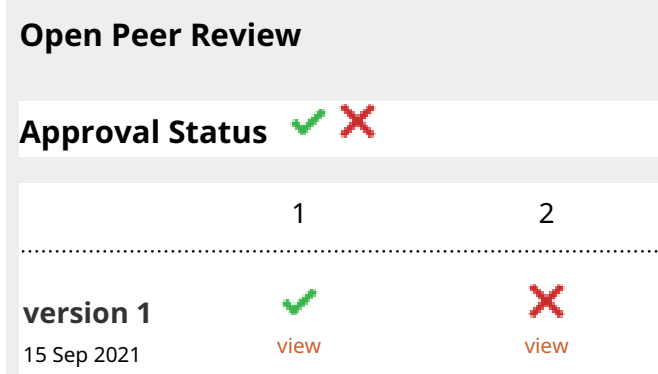

1. Quar Tian Kar, Universiti Kebangsaan Malaysia, Bangi, Malaysia

2. Lindsey E. Jorgensen, University of South Dakota, Vermillion, USA

Any reports and responses or comments on the article can be found at the end of the article. 
Corresponding author: Yasothei Suppiah (yasothei.suppiah@mmu.edu.my)

Author roles: Suppiah Y: Investigation, Writing - Review \& Editing; Maruthan MC: Formal Analysis, Investigation, Methodology; Abas FS: Supervision

Competing interests: No competing interests were disclosed.

Grant information: The author(s) declared that no grants were involved in supporting this work.

Copyright: ( 2021 Suppiah Y et al. This is an open access article distributed under the terms of the Creative Commons Attribution License, which permits unrestricted use, distribution, and reproduction in any medium, provided the original work is properly cited.

How to cite this article: Suppiah Y, Maruthan MC and Abas FS. Hearing aid through skin sensory for profound deaf people [version 1; peer review: 1 approved, 1 not approved] F1000Research 2021, 10:926 https://doi.org/10.12688/f1000research.72979.1

First published: 15 Sep 2021, 10:926 https://doi.org/10.12688/f1000research.72979.1 


\section{Introduction}

The World Health Organization (WHO) has adopted a grading system based on audiometric measurements to standardize the way in which severity of hearing loss of a person is reported. According to WHO, ${ }^{1}$ a profound deafness is defined as a person who has a hearing threshold of 80 to 95 decibels (dB). A person with complete or total hearing loss has a threshold of $95 \mathrm{~dB}$ or more. On the other hand, a normal hearing person has a hearing threshold of less than $20 \mathrm{~dB}$. Furthermore, they stated that almost 30 million people worldwide have profound or complete hearing loss in both ears. Based on their reported statistics on number of people with moderate or higher levels of hearing loss, $80 \%$ are from low income and middle-income countries as opposed to $20 \%$ that are from high-income countries.

A hearing aid or a cochlear implant is the common solution for induvial that are profoundly or severely deaf. ${ }^{2}$ The purpose of hearing aid is sound amplification; therefore, it is more suitable for mild hearing impairment. ${ }^{3,4}$ Besides that, the treatment based on cochlear implant is too costly and involves complex and risky surgery. In addition, hearing-impaired users still encounter substantial practical and social challenges with the use of this aid. At present, sign language ${ }^{5-7}$ and speech-reading ${ }^{8}$ are commonly applied in schools for the deaf.

Recent studies show that the human ear and the auditory nerve are not the only channel for gaining audio signals. ${ }^{10,11}$ There are several processes such as obtaining sound signals, translating voice signals to electrical signals, and producing frequency resolution can be achieved through the advancement of electronic devices. In other words, this additional tactile stimulus could help the profoundly deaf person wearing the electronic device to perceive sounds.

A study on multi-channel array skin-hearing technology was proposed which involved the stimulation of the skin by the electrical signal based on the sound. ${ }^{9}$ This enabled the profoundly deaf person to receive sound signals through the skin to accomplish the purpose of hearing the sound. The advantages of their technology were that it does not rely on intact hearing, and it was cost-effective. Furthermore, Li, J et al. ${ }^{10}$ showed that voice signal can be transferred using the cutaneous sensory nerves and it can also distinguish different speech signals. Their findings demonstrate that skin sensory nerves are a reliable replacement for the auditory nerve in the challenges facing individuals with severeto-profound hearing loss.

A very recent study ${ }^{11}$ has shown that these individuals can learn to identify sounds that are algorithmically translated into patterns of vibration on the skin on the wrist. Furthermore, the users of the device could identify different sounds from the vibrations alone, which gradually improved over the course of one month. The analysis on the wearable sensory device unlocks the gateway to auditory stimuli, which could be interpreted through skin. ${ }^{11}$

The aim of this study was to develop a hearing aid that stimulates "hearing" through skin sensory, which is more affordable and accessible for the profoundly deaf or total hearing loss community. Hence, we embarked into this research as studies on hearing through skin sensory seems to be a promising area for the beneficial of the society of profound hearing people in terms of safety and affordability.

\section{Methods}

The first phase of this study was to design a skin sensory stimulator by using a hand phone coin vibrator or micro actuator (MEMS) that allows the sound vibrations to be detected through the user's skin. Figure 1 briefly demonstrates the stages of designing the human sensory stimulator.

A Sound Transducer uses electrical energy to produce mechanical vibrations to disturb the surrounding air to produce sound regardless of whether the frequency is audible or inaudible. Audio Sound Transducer includes an input sensor that transforms sound into an electrical signal such as a microphone, and an output actuator that transforms the electrical signals back into sound, such as a loudspeaker. A preamplifier converts a weak electrical signal into an output signal, which is powerful enough to be noise tolerant. Absence of this will cause the output signal to be noisy or misleading. Due to this, the preamplifier is frequently located near to the sensor which reduces the effects of noise and other interference. A frequency filter is an electrical circuit that either stops or passes frequency from electrical signal with respect to specific frequency needed for further process. There are two types of filters, passive filter which uses passive components, and

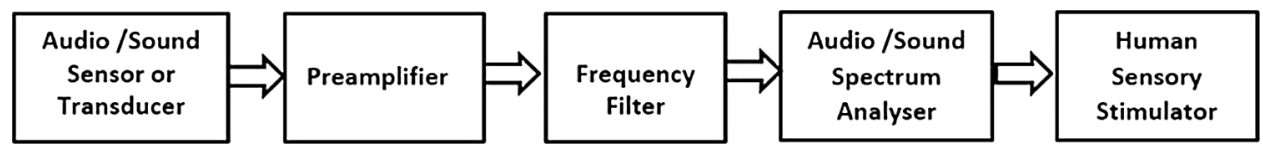

Figure 1. Flow design of human sensory stimulator. 
active filter that uses active components. Audio spectrum analysers are commonly used by sound engineers for various applications. Measuring the frequency response and analysing distortion characteristics of different types of audio tools are some of these applications. Micro actuators are active devices proficient of producing mechanical motion of solids or fluids. Hand phone coin vibrator is taken from the concept of cell phone vibration motor. We developed a simple single audio signal vibration array, which is based on the LM3915 Dot/Bar Display Driver and an electret microphone.

Sound sensor module gives a simple method to detect sound and it is usually used for the purpose of detecting sound intensity. It uses a microphone as a transducer which supplies the input audio signal to an amplifier, peak detector, and buffer. This module is made of three functional elements, the sensor on the front of the module will perform the pick-up (transducer), then the analogue signal is sent to the amplifier to amplify the signal. Accommodated with the adjustable voltage reference and an accurate ten-step voltage divider is the IC LM3915. The advantage of substituting conservative meters with a light-emitting diode (LED) bar graph is that it provides a quicker response, and a more rugged display with high visibility that provides the ease of interpretation of an analog display. As an initial test device, a transducer vibrating device circuit is designed to study the response of subjects to vibrating motor or actuator to skin sensory. The circuit (Figure 2) utilises a LM358 operational amplifier. An electret microphone preamplifier will pick-up the audible signal from the surrounding and amplifies it through a first stage operational amplifier to a process able signal. The signal will then pass through a second stage amplifier comparator to increase the voltage level high enough to drive the vibrator or actuator. A transistor interface is used to drive the vibrator or actuator in accordance with the sound received from the electret microphone.

In this research, the initial testing model was designed based on the LM3915 audio level indicator with LEDs converted to hand phone coin vibrating devices. The circuit design development and drawing were done with the NI Multisim Electronic computer-aided design (CAD) software. The circuit is then simulated to produce a printed circuit board (PCB). The schematic circuit diagram is shown in Figure 3. The initial design (Figure 3) will be upgraded to an audio spectrum analyser (Figure 4), by using the Arduino processor for a multichannel wearable spectrum vibrator.

The spectrum analyser measures and displays the amplitude of a given input signal set verses the full frequency ranges of the instrument. Its main purpose is to measure the strength of the spectrum of both known and unknown signals. This audio spectrum analyser enables us to see the frequencies present in audio or sound and produces the graph of all the frequencies that are present in a sound source at any given time. The Arduino is a microcontroller board that contains everything needed to support the microcontroller to create a led audio spectrum matrix. The board is equipped with sets of digital and analogue input/output (I/O) pins that may be interfaced to various expansion boards (shields) and other circuits.

The initial experiment uses a single overall sound to vibrate the device which will be used to stimulate the skin sensory. The electret microphone will pick up audio or sound signal which will feed to a preamplifier with adjustable gain of sound. This is to test the subject whether they can perceive sound through the vibrating motor or actuator. The single vibrating device then will be upgraded to Audio spectrum vibrators in respond to a visible led matrix. The led matrix

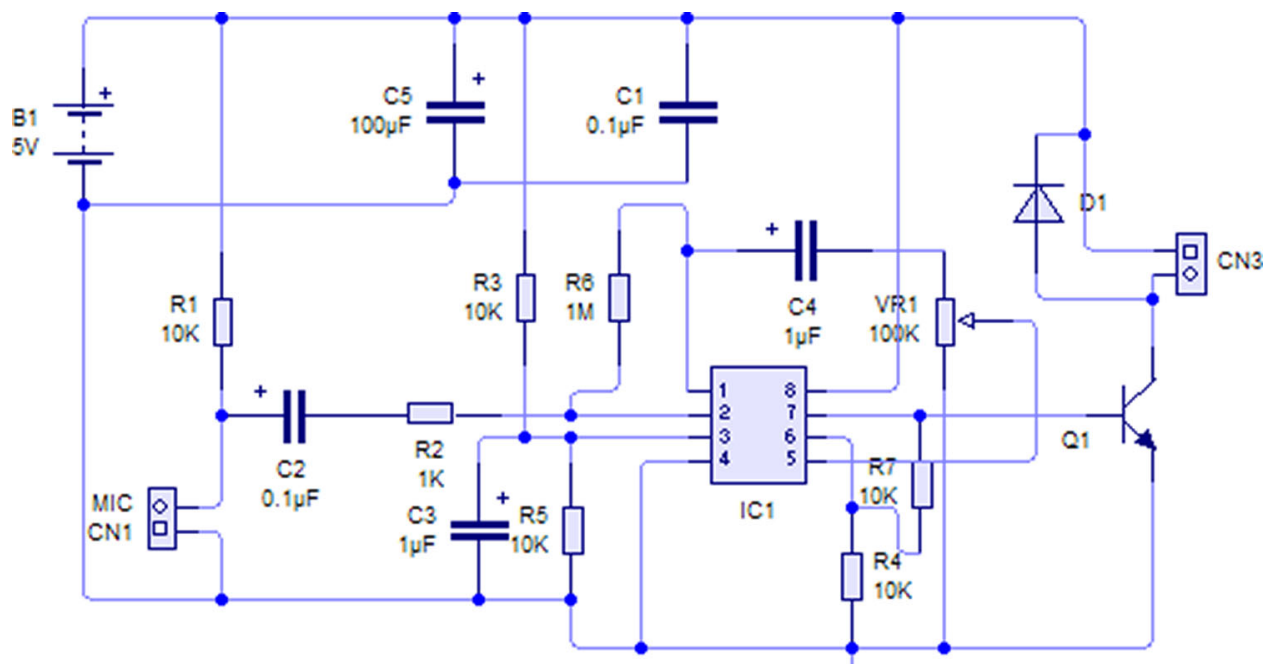

Figure 2. Single sound to vibration actuator driver. 


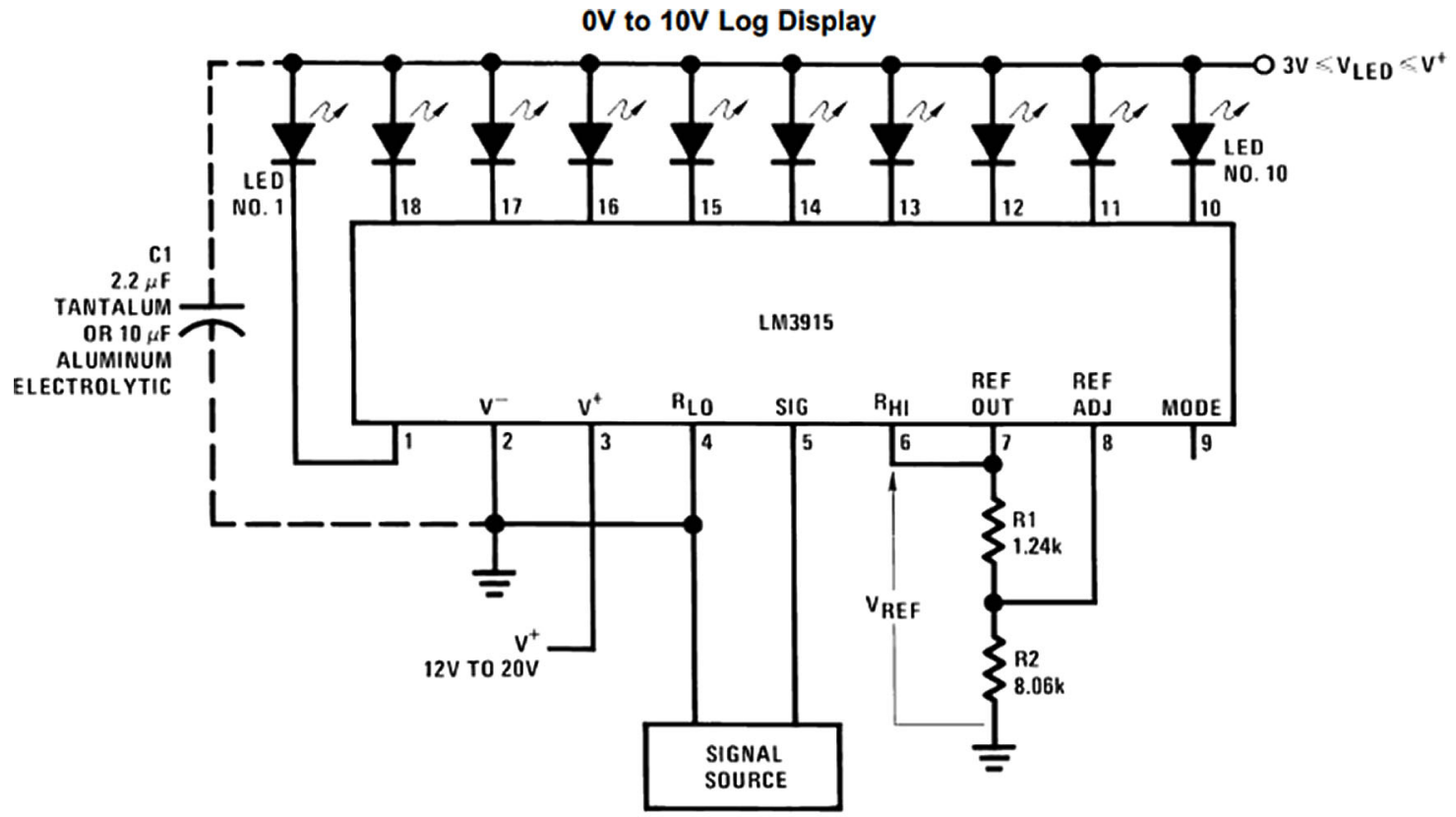

Figure 3. Schematic circuit diagram.

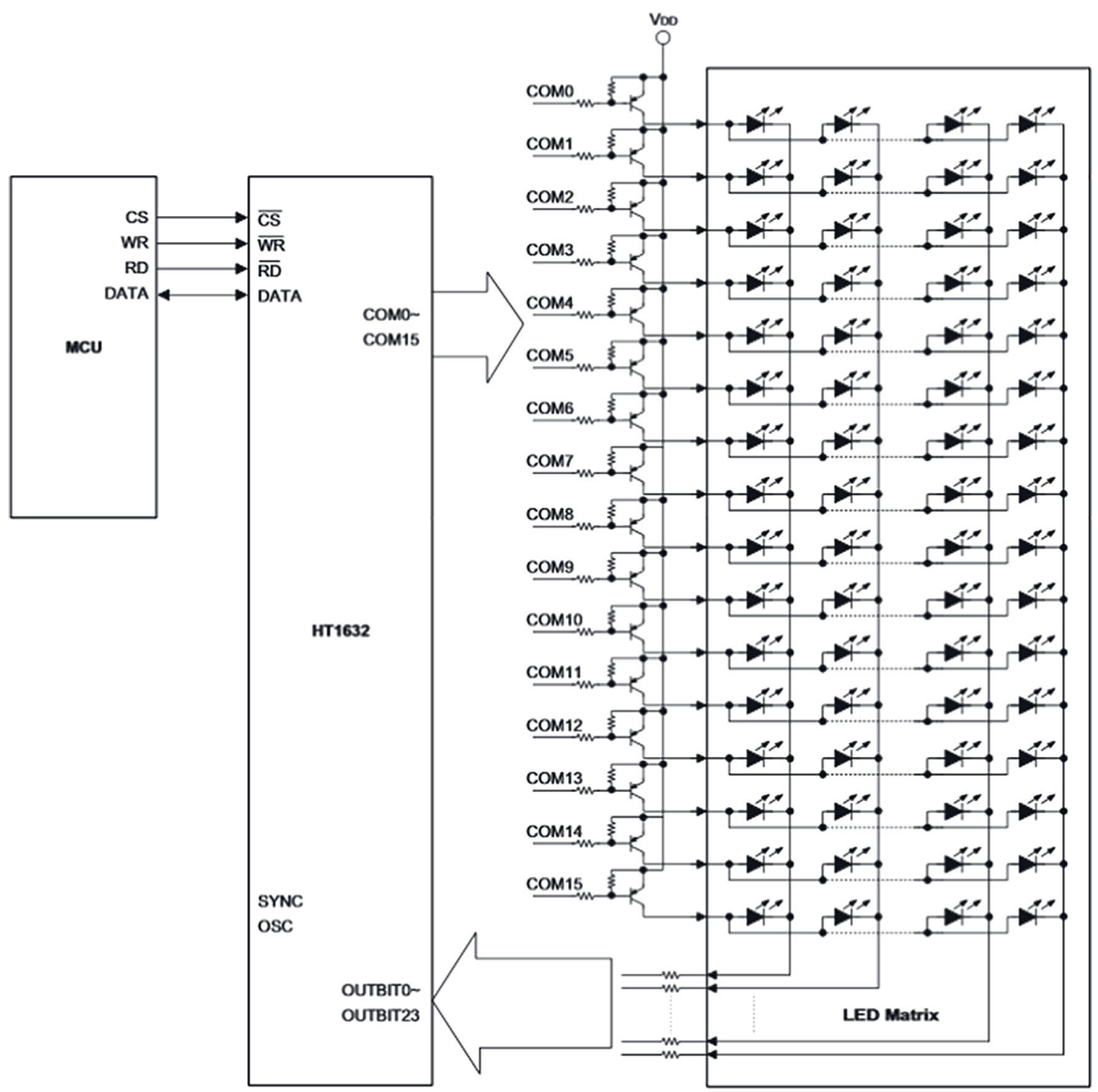




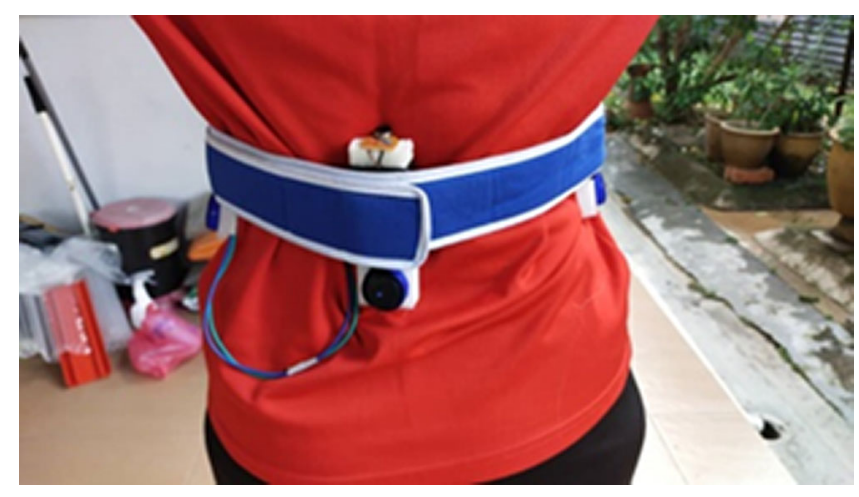

Figure 5. Belt strapped to subject's waist

would then be replaced with coin vibrator or actuator to give more precise vibrating pattern. The sound sensor can detect the frequency range from $20 \mathrm{hz}-20 \mathrm{khz}$ as this is the normal human hearing range. By using this type of sound sensor, it will enable the profoundly deaf people to "hear" the sound through their skin. The hand phone coin vibrators or micro actuator acts as an audio spectrum vibrator to create a vibrating pattern. This vibrating pattern will then be used to stimulate the human sensory system such as the skin for the profoundly deaf person, so they can use the vibration pattern as information. This repeating vibrating pattern stimulation will create a memory of a particular vibrating pattern for future recognition when similar sound is "heard" through the microphone.

The LED Matrix will have different types of frequency columns and its own vibrating devices with different sound intensity $(\mathrm{dB})$ levels. Each column will indicate the frequency level representation based on how it is programmed. For example, if there is a sound present, the frequency of the led matrix column will be activated to the maximum dB level which was picked up by the electret microphone. By changing the LEDs to vibrating stimulation, the profoundly deaf person can feel and "hear" the sound through his or her skin sensory system.

\section{Results}

Due to the recent outbreak of covid-19 pandemic, profoundly deaf individuals from different institutions, schools, or hospitals were not able to participate in our study.

Therefore, we randomly selected 30 people: 20 normal, seven partially deaf and three profoundly deaf (Underlying data) (12), who were mostly friends and relatives that lived within a $10 \mathrm{~km}$ radius of the authors residential area. All the 30 participants have given a written consent and they voluntarily agreed to participate in this study. We developed four initial vibrating transducers with single spectrum, which were strapped to a belt. Once the individuals wore the belt on his/her waist, the four transducers were placed at the front, back, left, and right side of the individual's waist (Figure 5). Audible sound was played (stimulus) and the individual was asked whether he/she felt a stimulation at the point of the transducer and if so, state the direction of the sound source. Based on the experiments, both normal and the partial or profoundly deaf individuals felt the vibrating stimulation on their skin and accurately stated the directions of the sounds. This initial experiment was successful, which is an important direction for the future development of a hearing aid through human sensory skin. Further advancement of a more comprehensive device using the concept of spectrum analyser is now in progress. The sound signals will then be fed to multiple audio filters and audio spectrum analyser to produce sound audio spectrum to drive multiple vibration on motors or actuators of the device.

\section{Conclusions}

Despite the advancement of various hearing devices that improve the life of profoundly deaf people around the globe, there are still many limitations and drawbacks, which emphasizes the need to further improve such technologies. The study of "hearing through the skin" is very promising and leads to affordable and safe solutions for the profoundly deaf and completely deaf people. As far as we are aware, adopting the concept of audio spectrum analyser for developing hearing aid has not been previously studied. By wearing the device, a profoundly deaf person can perceive sound through their skin. Future studies should investigate and address how various types of sound stimulated through the skin by devices such as the belt used in this study, can be interpretated and identified by the the profoundly deaf individual. 


\section{Ethical approval}

The Research Ethics committee of Technology Transfer Office, Multimedia University has granted ethics approval for this study. Ethical Approval Number: EA1992021. All participants of this study gave informed written consent to take part in the research.

\section{Data availability}

Underlying data

Open Science Framework (OSF): Hearing aid through skin sensory for profoundly deaf people.

\section{DOI: $10.17605 / O S F . I O / M H W F P .{ }^{12}$}

This project contains the following underlying data:

Data file. Data contains the participants' age, sex, hearing level, and ability to feel the vibration of the belt.

Data are available under the terms of the Creative Commons Zero "No rights reserved" data waiver (CC0 1.0 Public domain dedication).

\section{Author contributions}

Yasothei Suppiah: Conceptualization, investigation, supervision. M Chandran Maruthan: investigation, methodology, resources. Fazly Salleh Abas: validation, visualization, writing-original draft.

1. World Health Organization (WHO): World report on hearing. 2021. Reference Source

2. Zhang $M, G a o Y$ : Hearing level of deaf children in deafmute schools. Zhonghua Erbiyanhou Toujing Waike Zazhi (Chinese Journal of Otorhinolaryngology Head and Neck Surgery). 2008; 15: 671-673.

3. Li JW, Tang T: Applications in the Skin-Hearing Aid Compact by Embedded Technique. Microelectronics Computer. 2009; 5: 239-241.

4. Cui J, Xiao L, Wang $Y$, et al.: A kind of design criterion for WOLA filterbanks used in digital hearing aids. Yingyong Shengxue. 2010; 29(1): 36-42. Publisher Full Text

5. Du QL, Zhu ZW: Sign language in special art education. J Changchun University. 2010; 2: 81-82.

6. Jiang $\mathrm{HQ}$, Pan $\mathrm{H}$ : Key frame based multi-level classification of sign language recognition. Jisuanji Yingyong Yanjiu. 2010; 2 491-493.
7. Zheng $\mathrm{X}$ : Lingual status of Chinese finger language. Tinglixue $\mathrm{ji}$ Yanyu Jibing Zazhi. 2010; 1: 57-58.

8. Yan W: An exploration of round-mouth characters of zhi- system read in labiodental sound in shandong. Shandong Daxue Xuebao Zhexue he Shehui kexue U Shandong University (Philosophy and Social Sciences)). 2006; 2: 151-155.

9. Li JW, Liu W, Han XJ: Response of skin to audible signals. Shengxue Jishu. 2006; 25(3): 253-257.

10. Li J, Li Y, Zhang $M$, et al.: Cutaneous sensory nerve as a substitute for auditory nerve in solving deaf- mutes' hearing problem: an innovation in multi-channel- array skin-hearing technology. Neural Regeneration Res. 2014; 9(16): 1532-1540. PubMed Abstract | Publisher Full Text | Free Full Text

11. Michael VP, Thorhildur A, David ME: Deciphering Sounds Through Patterns of Vibration on the Skin. Neuroscience. 2021; 458, 77-86. Publisher Full Text

12. Suppiah Y: Data for hearing aid through skin sensory. 2021 Publisher Full Text 


\section{Open Peer Review}

\section{Current Peer Review Status:}

\section{Version 1}

Reviewer Report 17 January 2022

https://doi.org/10.5256/f1000research.76596.r99668

(C) 2022 Jorgensen $\mathbf{L}$. This is an open access peer review report distributed under the terms of the Creative Commons Attribution License, which permits unrestricted use, distribution, and reproduction in any medium, provided the original work is properly cited.

\section{Lindsey E. Jorgensen}

Department of Communication Sciences and Disorders, University of South Dakota, Vermillion, SD, USA

This is an interesting study and the background is very good. However, the methods lack significantly.

1. It is not made clear how the signal was processed, and this causes issues with replication. The authors should include frequency, ranges of frequencies, and intensity. What filtering did they use in the FFT?

2. While it is understood that COVID was a factor, it is of concern that the person was able to "hear" while also feeling, given that the subjects were not hard of hearing. Therefore, they could also hear the signal and not just feel it. This is difficult for validation.

3. The authors also did not demonstrate how the person's response was intended, and did not give a criteria by which the subjects response was to be measured? (awareness, perception, recognition, etc).

Is the work clearly and accurately presented and does it cite the current literature? Yes

Is the study design appropriate and is the work technically sound? Partly

Are sufficient details of methods and analysis provided to allow replication by others? No

If applicable, is the statistical analysis and its interpretation appropriate? No 
Are all the source data underlying the results available to ensure full reproducibility? No

Are the conclusions drawn adequately supported by the results?

Partly

Competing Interests: No competing interests were disclosed.

I confirm that I have read this submission and believe that I have an appropriate level of expertise to state that I do not consider it to be of an acceptable scientific standard, for reasons outlined above.

Reviewer Report 19 October 2021

https://doi.org/10.5256/f1000research.76596.r96595

(c) 2021 Tian Kar Q. This is an open access peer review report distributed under the terms of the Creative Commons Attribution License, which permits unrestricted use, distribution, and reproduction in any medium, provided the original work is properly cited.

\section{Quar Tian Kar}

Audiology Program, Centre for Rehabilitation and Special Needs Studies, Faculty of Health Sciences, Universiti Kebangsaan Malaysia, Bangi, Malaysia

The study developed a device that converts sound patterns into patterns of vibrotactile stimulation. Overall, the article is well written, and the information presented in the article is well explained, organized and relevant to the study. The ideas are well-organized and coherent, making it easy for readers to follow and understand. This is an interesting study as it explains the development of a sensory device that has the potential to provide benefits to people diagnosed with profound hearing loss that cannot be aided either with conventional hearing aids or cochlear implant. The research design is appropriate, adequate, and novel in the sense it was developed by the researchers and validated.

Some comments:

In the abstract, the second sentence mentions a hearing aid's "ineffectiveness" for those who are profoundly deaf. This is perhaps not accurate and should not be generalized to all individuals with hearing loss. With the advancement of technology, today's hearing aids are found to be useful even for profoundly deaf people. Perhaps it should be replaced with 'limitations' of hearing aids.

In the second paragraph of the Introduction section, it would be good if the authors can elaborate a bit more why hearing aids are suitable only for mild hearing loss and not helpful for other degrees of hearing loss. "Individual" is misspelled in the first sentence.

There are few comments from me on the results section where it would be good if the authors could elaborate a bit more, so that readers can have a better understanding. It was mentioned 
that 30 subjects were recruited for the study. Seven were partially deaf. Please explain the meaning of 'partially deaf'. Please explain the purpose of recruiting subjects with different degrees of hearing loss. Would individuals with different degrees of loss have different experiences of the vibration stimulation? The reader might be interested to know what kind of sound (stimulus) was used in the experiment and how the stimulus contributed to the ability of the subjects to tell the direction of the sound source.

It will be interesting for future studies to explore the possibilities of developing what the authors have suggested, the concept of audio spectrum analyzer that enables profoundly deaf persons to interpret sounds through tactile hearing aids.

Is the work clearly and accurately presented and does it cite the current literature? Yes

Is the study design appropriate and is the work technically sound?

Yes

Are sufficient details of methods and analysis provided to allow replication by others? Partly

If applicable, is the statistical analysis and its interpretation appropriate? Yes

Are all the source data underlying the results available to ensure full reproducibility? No source data required

Are the conclusions drawn adequately supported by the results?

Yes

Competing Interests: No competing interests were disclosed.

Reviewer Expertise: I am an expert in Audiology, a branch of science that studies hearing, balance and related disorders. My research interest include hearing aid evaluation and validation. The technical aspects of this research is beyond my expertise.

I confirm that I have read this submission and believe that I have an appropriate level of expertise to confirm that it is of an acceptable scientific standard. 
The benefits of publishing with F1000Research:

- Your article is published within days, with no editorial bias

- You can publish traditional articles, null/negative results, case reports, data notes and more

- The peer review process is transparent and collaborative

- Your article is indexed in PubMed after passing peer review

- Dedicated customer support at every stage

For pre-submission enquiries, contact research@f1000.com 